\title{
Scanning Microscopy
}

$11-2-1992$

\section{Time-Resolved Scanning Electron Microscopy Analysis of Nanodynamical Structures}

\author{
J. Jason Yao \\ Cornell University, Ithaca
}

Noel C. MacDonald

Cornell University, Ithaca

Follow this and additional works at: https://digitalcommons.usu.edu/microscopy

Part of the Biology Commons

\section{Recommended Citation}

Yao, J. Jason and MacDonald, Noel C. (1992) "Time-Resolved Scanning Electron Microscopy Analysis of Nanodynamical Structures," Scanning Microscopy. Vol. 6 : No. 4 , Article 5.

Available at: https://digitalcommons.usu.edu/microscopy/vol6/iss $4 / 5$

This Article is brought to you for free and open access by the Western Dairy Center at DigitalCommons@USU. It has been accepted for inclusion in Scanning Microscopy by an authorized administrator of DigitalCommons@USU. For more information, please contact digitalcommons@usu.edu.

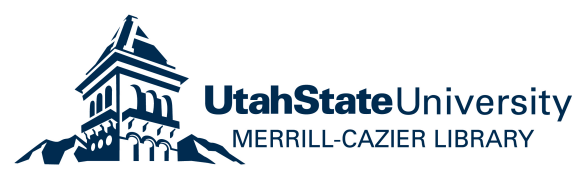




\title{
TIME-RESOLVED SCANNING ELECTRON MICROSCOPY ANALYSIS OF NANODYNAMICAL STRUCTURES
}

\author{
J. Jason Yao and Noel C. MacDonald*
}

School of Electrical Engineering and the National Nanofabrication Facility Cornell University, Ithaca, NY 14853

(Received for publication July 20, 1992, and in revised form November 2, 1992)

\begin{abstract}
We have developed and characterized a timeresolved scanning electron microscopy (SEM) operational mode which provides non-destructive mechanical characterization of nanodynamical structures with 8-bit image resolution and $200 \mathrm{~ns}$ time resolution. This time-resolved SEM scheme does not require blanking plates, nor does it require any hardware modification to a commercially available scanning electron microscope. Both timeresolved images and line scan profiles of nanofabricated single crystal silicon tweezers are obtained. This time-resolved SEM operational mode can be used to evaluate a number of important mechanical properties of nanodynamical structures, including time response and resonance mode-shapes.
\end{abstract}

KEY WORDS: Time-resolved analysis, nanodynamical structure, microdynamics, microelectromechanical systems (MEMS), nanofabrication, microfabrication, micromechanics, non-destructive mechanical characterization, time response, resonance mode-shapes.

*Address for correspondence:

Noel C. MacDonald

Department of Electrical Engineering

Cornell University

Ithaca, NY 14853

Phone No.

(607) 255-3388

\section{Introduction}

One key advantage of the growing field of microdynamics or microelectromechanical systems (MEMS) is its capability to improve performance, density and cost reduction through down-scaling of mechanical systems in size. Batch fabricated microstructures with low mass, high stiffness, and small thermal mass have been realized. These microstructures exhibit many advantageous properties as compared with their macroscopic counterparts. Examples range from advanced stateof-the-art sensor devices (Kenny et al., 1991), to microfabricated side-drive motors (Mehregany et al., 1992), to a scanning tunneling microscope (STM) on a chip (Akamine et al., 1989). We are further enhancing these properties of MEMS by down-scaling the structures in size to achieve "nanodynamical structures." Nanoscale, single crystal silicon scanned probe sensor devices have thus been fabricated and reported (Yao et al., 1991; Yao et al., 1992).

The electromechanical characterization of microdynamical structures offers new challenges. A typical nanometer-scale, high speed structure has feature sizes on the order of one nanometer to a few hundred nanometers and a mechanical resonant frequency of the order of $5 \mathrm{MHz}$ (Yao et al., 1991; Yao et al., 1992). This range of dimensions is too small to be resolved by a conventional optical microscope due to diffraction-limitations, and the speed of the mechanical motion provided by this type of nanostructure exceeds the scanning rate of a scanning electron microscope (typically $30 \mathrm{~Hz}$ ); thus the details of the motion cannot be captured by normal scanning electron microscopy (SEM) images. Figure 1 shows two SEM images of a simple nanometer-scale, tweezers-shaped (MacDonald et al., 1989), single crystal silicon (SCS) structure with a nominal beam width for the tweezers of $250 \mathrm{~nm}$. When a potential difference is applied between the two beams of the tweezers, an electric field-induced force produces an attractive dynamical motion of the beams. Figure 1(a) is a SEM image of the SCS tweezers with no applied voltage. Figure $1(\mathrm{~b})$ is the next SEM image recorded after a step function voltage is applied to the SCS 


\section{J. J. Yao and N. C. MacDonald}
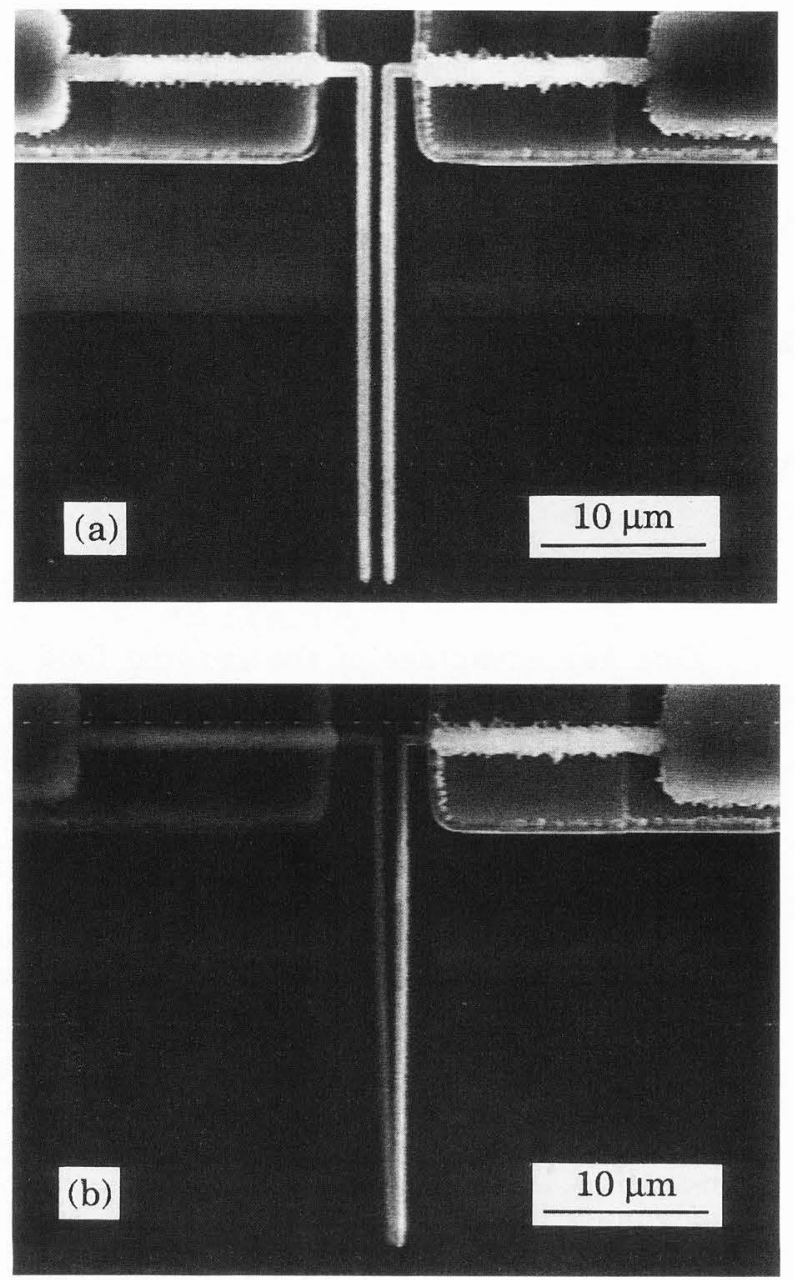

Fig. 1. SEM images of the SCS tweezers (a) with no voltage applied, and (b) after a step function voltage is applied.

tweezers. By examining the two images, it is apparent that the time response of the SCS tweezers to the step function voltage is not recorded by TVrate $\mathrm{SEM}$ imaging.

Time-resolved and stroboscopic scanning microscopy (MacDonald et al., 1969; Plows and Nixon, 1968) have been used to investigate microelectronic circuits (Feuerbaum, 1979). Time varying SEM characterization based on negative electron affinity photocathodes has also been previously reported with temporal resolution as high as 100 ps (Sanford and MacDonald, 1989). We report a time-resolved SEM characterization scheme that does not require blanking plates, for mechanical characterization of nanodynamical structures. This time-resolved scheme is capable of evaluating a number of dynamical structural properties including time response and resonance mode-shapes, which are essential properties of a mechanical system. Line scan images and stroboscopic micrograph images of nanodynamical structures have been obtained using this timeresolved scheme. In this paper, we describe the experimental setup and present the time-resolved SEM results.

\section{Experimental Setup}

The time-resolved SEM experiment is performed in a Cambridge S200 (Cambridge Inc., Cambridge, England) at the National Nanofabrication Facility (NNF), Cornell University. No modifications are made to the SEM to ensure that the usual SEM functions remain unchanged for other users at the facility. Figure 2 is a schematic diagram of the experimental setup for the timeresolved SEM study. The pulse generator (HP 3183, Hewlett Packard Co.) is operated in the burst mode. For every trigger signal produced by the SEM's line synchronization generator, the pulse generator sends out $n$ number of pixel pulses. The number $n$ is set to be equal to the number of pixels per line in the video acquisition system. In this experiment, $n$ $=512$. The pixel pulses are sent to a programmable pulse counter. This pulse counter counts the number of pixel pulses starting from the preset count; sends out a step function when zero count is reached; and is reset at the end of a SEM scanning line, i.e., during the SEM line retraces. This step function is then amplified to the necessary amplitude to actuate the SCS tweezers. The SEM

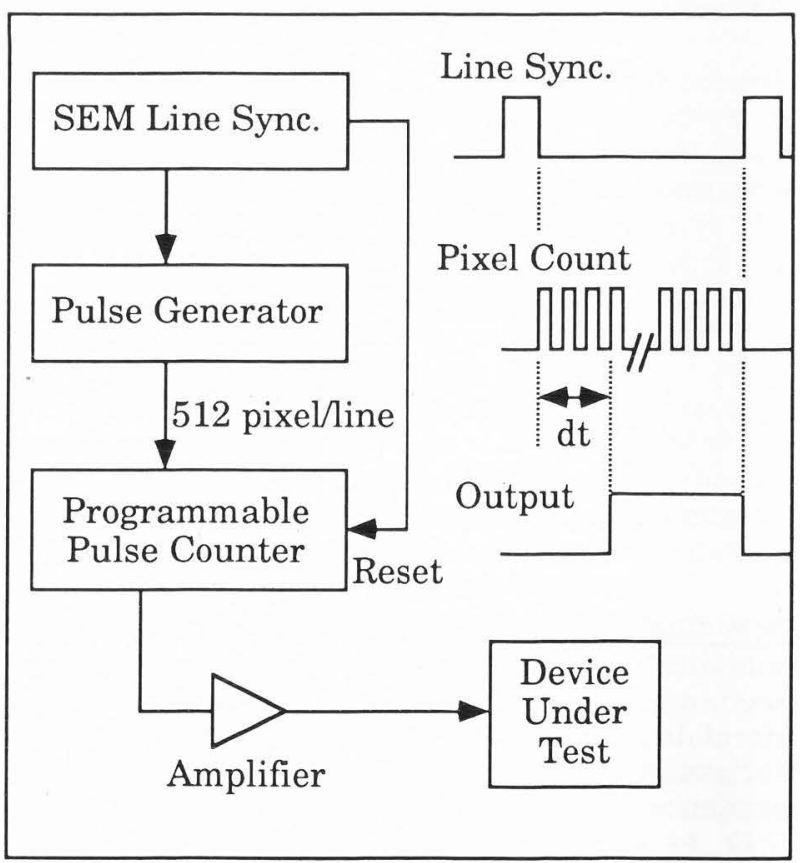

Fig, 2. Schematic diagram of the experimental setup for the time-resolved SEM analysis. 
video images of the SCS tweezers with all combinations of the preset count of the programmable pulse counter are digitized and stored for later processing.

The SCS tweezers shown in Fig. 1 is used to demonstrate the time-resolved SEM operational mode. Figure 3 shows three SEM images of the SCS tweezers. The number shown at the upper-right corner of each image is the preset count of the programmable pulse counter. Therefore, in Fig. $3(\mathrm{a})$, there are 40 pixels skipped at the beginning of each line of the image before the step function voltage is applied to the SCS tweezers. Similarly, 68 pixels and 122 pixels are skipped in Fig. 3(b) and Fig. 3(c), respectively. Note that the step function is triggered at least one "tweezers-response-time" before the scanning electron-beam of the SEM scans over the SCS tweezers in Fig. 3(a), thus the SEM image shows the tweezers in the closed position. In Fig. 3(b), the step function is turned on after the SEM images a portion of the left beam of the tweezers, thus the image shows a portion of the left beam of the tweezers in the open position and the rest of the tweezers in the closed position. Lastly, in Fig. 3(c), the step function rises after the scanning electron-beam of the SEM passes the SCS tweezers, thus the SEM image shows the tweezers in the open position.

To illustrate the post-processing of the video images for obtaining time-resolved images, we examine a simplified example of the video imaging system shown in Fig. 4. This simplified imaging system is constructed with 5 pixels per line and 5 lines per frame as indicated in Fig. 4(a). Figures 4(b) to 4(d) are three images acquired with the preset count of the programmable pulse counter being 0 to 2 , respectively. Therefore, column 3 in Fig. 4(c), for example, contains the video information about location column 3 with a onepixel-period delay. Similarly, column 4 in Fig. 4(b) contains the video information about location column 4 with a three-pixel-periods delay. Thus, to reconstruct a $5 \times 2$ time-resolved image at location columns 3 and 4 from this simplified imaging system, column 3 from Fig. 4(c) and column 4 from Fig. 4(d) give an image with a one-pixel-period delay, and column 3 from Fig. 4(b) and column 4 from Fig. 4(c) give an image with a two-pixelperiods delay, and so on. Electron-beam shifting in the SEM due to sample biasing is not a factor in this time-resolved setup because all images are obtained after the step function is applied to the SCS tweezers, i.e., the sample surface potential is constant.

\section{Time-Resolved SEM Results}

Using the above time-resolved SEM setup and post image processing, Fig. 5 is a series of timeresolved images of the SCS tweezers responding to a step function of -45 Volts. In this actual imaging system, each frame contains 480 lines and each line contains 512 pixels (i.e., 480 x 512). To reconstruct
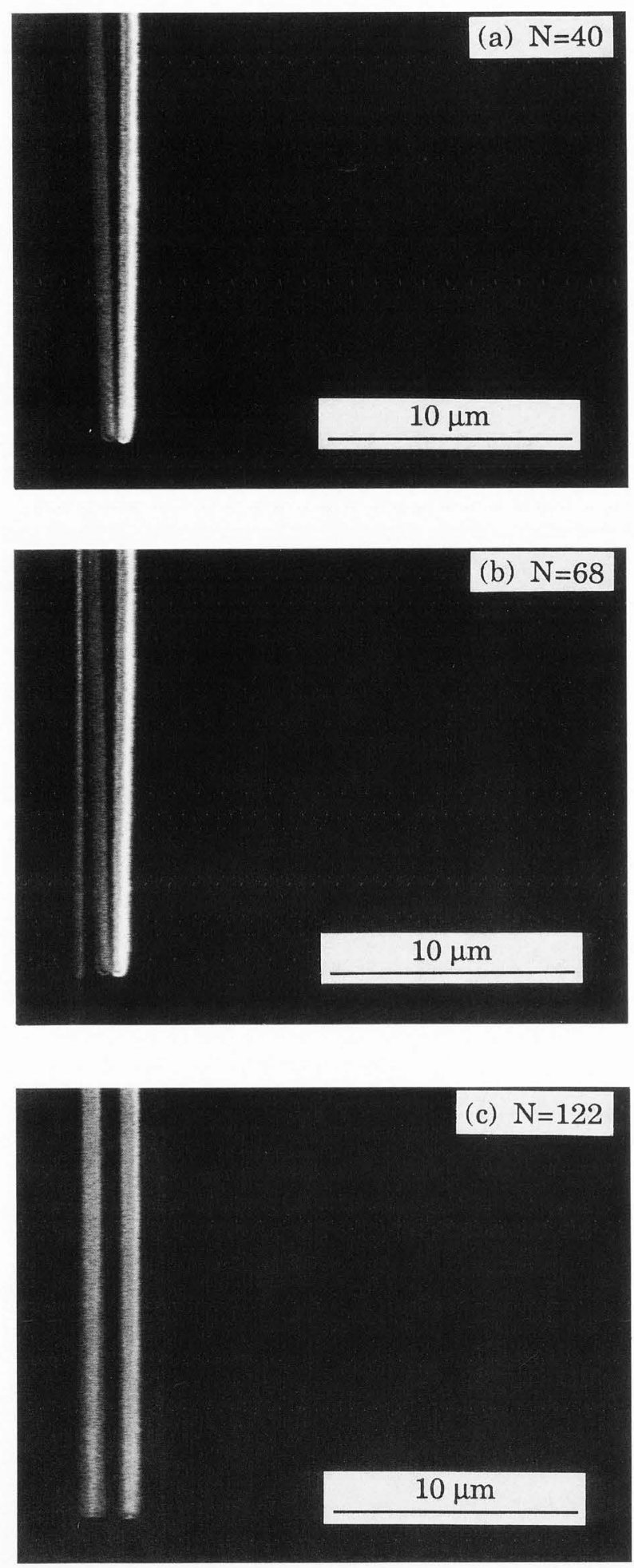

Fig. 3. SEM images of the SCS tweezers with the preset count of the programmable pulse counter set to (a) 40 , (b) 68 , and (c) 122 pixels. 


\begin{tabular}{|c|c|c|c|c|}
\multicolumn{1}{c}{1} & \multicolumn{1}{c}{2} & \multicolumn{1}{c}{4} & \multicolumn{1}{c}{5} \\
\hline p11 & $p 12$ & $p 13$ & $p 14$ & $p 15$ \\
\hline p21 & $p 22$ & $p 23$ & $p 24$ & $p 25$ \\
\hline p31 & $p 32$ & $p 33$ & $p 34$ & $p 35$ \\
\hline p41 & $p 42$ & $p 43$ & $p 44$ & $p 45$ \\
\hline p51 & $p 52$ & $p 53$ & $p 54$ & $p 55$ \\
\hline
\end{tabular}

(a)

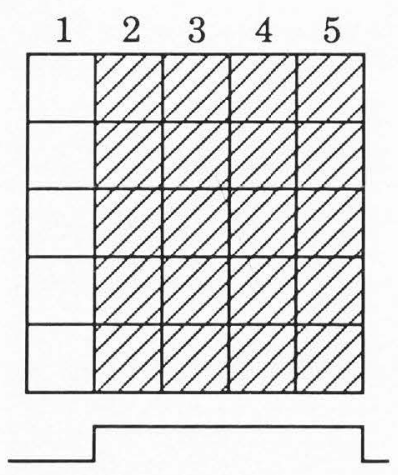

(c)

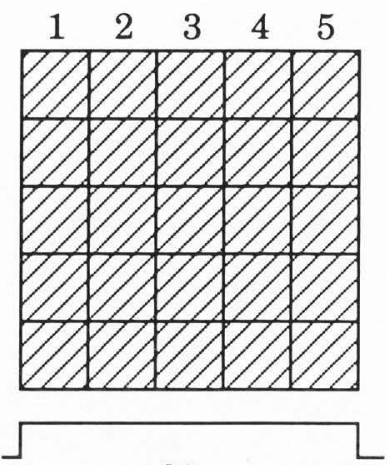

(b)

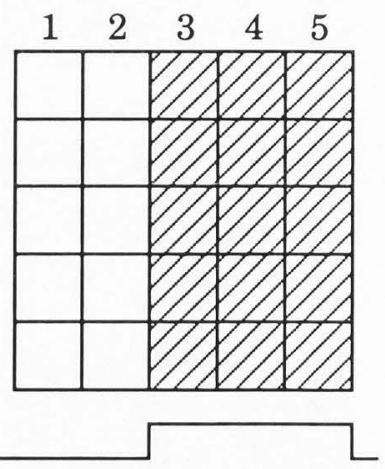

(d)

Fig. 4. An illustration of a simplified time-resolved video imaging system with (a) a 5 x 5 pixel array and a preset pixel count of the programmable pulse counter set to (b) 0 , (c) 1 , and (d) 2 pixels.

m number of $480 \times 120$ time-resolved images (e.g., images in Fig. 5), a total of $120+\mathrm{m}$ images ( $480 \times 512$ ) are digitized and stored with the preset count of the programmable pulse counter being varied from 1 to $120+\mathrm{m}$, sequentially. The pixel period is set to 860 ns by the pulse generator to match the pixel scan rate of the SEM (440 $\mu \mathrm{s} / 512$ pixels); therefore, the time increment between adjacent time-resolved images is $860 \mathrm{~ns}$. As indicated in Fig. 5, the SCS tweezers close within 5 adjacent images, i.e., within $4.3 \mu \mathrm{s}$, and the tweezers step response is a non-linear response with increasing rate of motion which is in qualitative agreement with previously reported numerical simulations of tungstentweezers (MacDonald et al., 1989).

Line scan profiles of the time-resolved images may also be readily extracted from the stored timeresolved data. Figure 6 is a series of line scan profiles of the SCS tweezers at the 360th line from the top (out of a total of 480 lines) with arbitrary video intensity units versus pixel counts zoomed in near the beams of the tweezers. These line scan profiles provide a more quantitative analysis of the SCS tweezers time response. Note that the difference between the two intensity peaks of the beams of the tweezers is an indication of the SEM voltage contrast due to the voltage difference between the two beams of the tweezers.

Both the time-resolved images and the line scan profiles have 8-bit resolution which is mainly determined by the performance of the SEM and of the video data acquisition system. The time resolution of this time-resolved SEM setup is determined by the bandwidth of the SEM video processor board and the pixel scan-rate because a pulse generator usually has better time resolution with lower trigger jitter than the SEM electronics. In this time-resolved SEM setup, the time
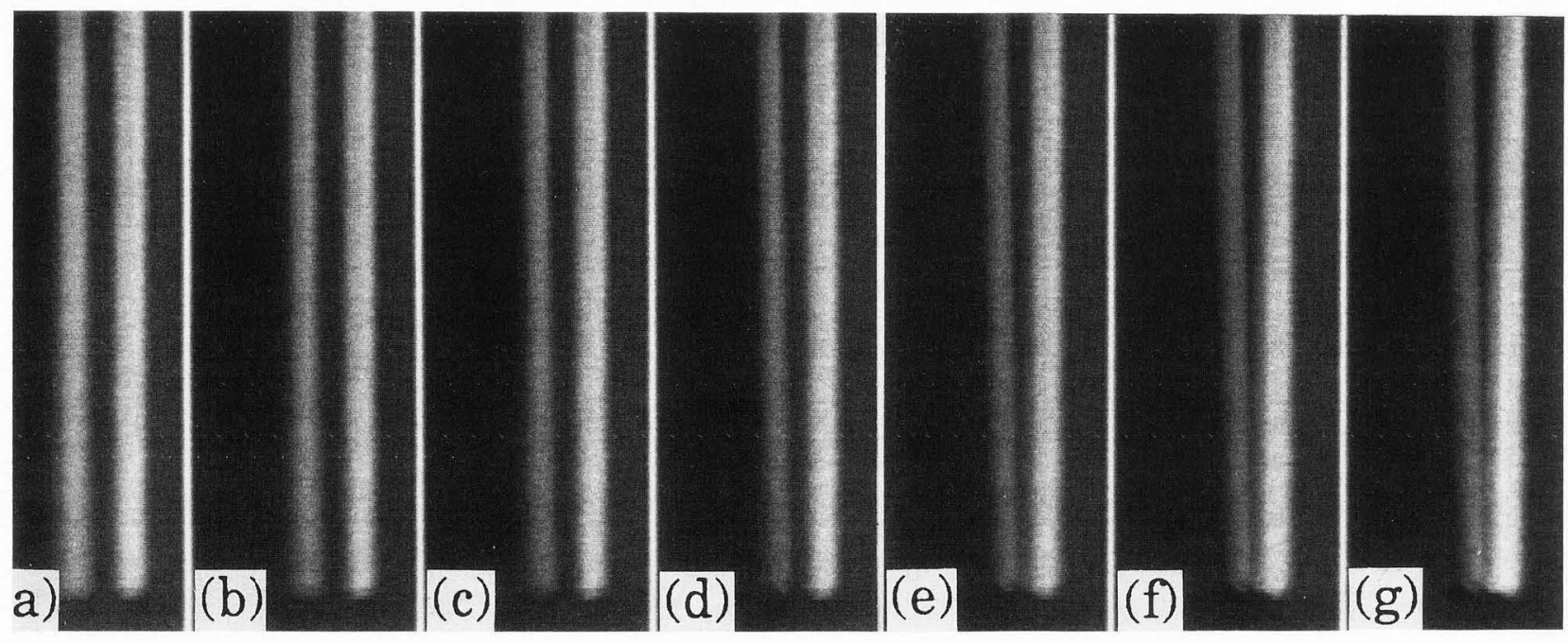

Fig. 5. A series of the time-resolved SEM images showing the response of the SCS tweezers to an applied step function of $-45 \mathrm{~V}$. The time increment between the images is $860 \mathrm{~ns}$. 


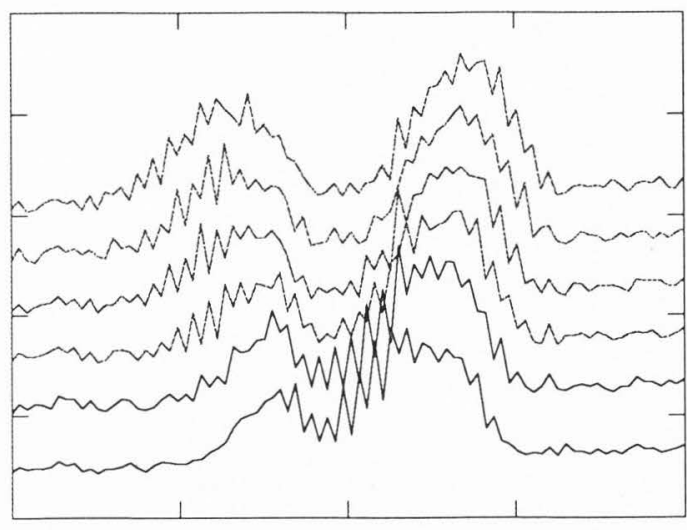

\section{Pixel Counts}

Fig. 6. A series of the time-resolved line scan profiles of the SCS tweezers at the 360 th line from the top of the time-resolved images illustrated in Fig. 5 (out of a total of 480 lines). The line scan profile was positioned near the end of the beams of the tweezers.

resolution is on the order of $200 \mathrm{~ns}$ while the pulse generator provides a $10 \mathrm{ps}$ resolution.

\section{Conclusions}

We have developed and characterized timeresolved SEM operational mode which provides nondestructive mechanical analysis of nanodynamical structures. This time-resolved SEM operation requires no modification within a commercially available SEM, and has 8-bit video resolution and a nominal $200 \mathrm{~ns}$ time resolution.

Nanofabricated single crystal silicon tweezers have been used to characterize this time-resolved SEM scheme. Both time-resolved SEM images and line scan profiles have been obtained, and show excellent agreement with the simulated results. This time-resolved SEM scheme can be used to evaluate a number of important mechanical properties of nanodynamical structures, including time response and resonance mode-shapes.

\section{Acknowledgements}

This work was supported by the National Science Foundation under Grant Nos. ECS-8805866 and ECS-8815775. The structures were fabricated at the National Nanofabrication Facility (NNF) which is supported by the National Science Foundation, Cornell University, and Industrial Affiliates. The authors would like to thank the staff of NNF for their technical assistance. The authors would also like to express their appreciation for the technical assistance provided by Tom H. Soh, Susanne C. Arney, and Wolfgang Hofmann.

\section{$\underline{\text { References }}$}

Akamine S, Albrecht TR, Zdeblick MJ, Quate CF (1989) Microfabricated scanning tunneling microscope. IEEE Electron Dev. Lett. 10 (11), 490492.

Feuerbaum HP (1979) VLSI testing using the electron probe. Scanning Electron Microsc. 1979; I: 285-296.

Kenny TW, Waltman SB, Reynolds JK, Kaiser WJ (1991) Micromachined silicon tunnel sensor for motion detection. Appl. Phys. Lett. $\underline{58}$ (1), 100-102.

MacDonald NC, Chen LY, Yao JJ, Zhang ZL, McMillan JA, Thomas DC, Haselton KR (1989) Selective chemical vapor deposition of tungsten for microelectromechanical structures. Sensors and Actuators 20, 123-133.

MacDonald NC, Robinson RM, White RM (1969) Time-resolved scanning electron microscopy and its application to bulk-effect oscillators. J. Appl. Phys. 40 (11), 4516-4528.

Mehregany M, Senturia SD, Lang JH, Nagarkar P, (1992) Micromotor fabrication. IEEE Trans. on Electron Devices 39 (9), 2060-2069.

Plows GS, Nixon WC (1968) Stroboscopic scanning electron microscopy. J. Phys. E 1, 595-600.

Sanford CA, MacDonald NC (1989) Laser pulsed GaAs cathodes for electron microscopy. J. Vac. Sci. Technol. B7 (6), 1903-1907.

Yao JJ, MacDonald NC, Arney SC (1991) Nanostructures in motion. In: Nanostructures and Mesoscopic Systems. Kirk W, Reed M (eds), Academic Press, New York, 25-33.

Yao JJ, Arney SC, MacDonald NC (1992) Fabrication of high frequency two-dimensional nanoactuators for scanned probe devices. IEEE J. Microelectromechanical Systems, 1 (1), 14-22.

\section{Discussion with Reviewers}

A. Gopinath: The method utilizes step incremental voltages. If the usual single step voltage of $-45 \mathrm{~V}$ causes bounce, this technique will not resolve this motion. Can the authors suggest modifications or alternative techniques (apart from true stroboscopy) to image this kind of motion?

Authors: The time-resolved SEM operational mode described in this paper has a temporal resolution of $200 \mathrm{~ns}$. If the mechanical bounce of the structure in a step response is much slower than $200 \mathrm{~ns}$, this time-resolved technique is in fact capable of resolving the bounce-motion. For structures with higher natural frequencies, i.e., faster bouncemotion, techniques with larger bandwidth are required (see answer to T.W. Kenny's question below).

T.W. Kenny: Is it possible to increase the pixel scan rate of the SEM to allow time-resolved observation of sub-us phenomena?

Authors: The temporal resolution of the timeresolved SEM operational mode is determined mainly by the scan-rate of the SEM and the 


\section{J. J. Yao and N. C. MacDonald}

bandwidth of the SEM's detector and video board. With hardware modifications to the SEM (the scan generator, the detector, and the video board), it is possible to achieve better temporal resolution to observe sub- $\mu$ s phenomena, with the upper limit of bandwidth being a few hundred megahertz. A very fast scan-rate would be required (5 ns/pixel). Temporal resolution higher than that could be achieved with other methods such as the one illustrated in Sanford and MacDonald (1989) (100 ps). 